\title{
BMJ Open Subsidy programme for gestational diabetes mellitus screening and lifestyle management in rural areas of western China: a study protocol for a multicentre randomised controlled trial
}

\author{
Tingting Xu (D) , ${ }^{1,2}$ Xiaozhen Lai, ${ }^{2}$ Kun He, ${ }^{2}$ Liangkun Ma (D) , ${ }^{3}$ Hai Fang ${ }^{2,4}$
}

To cite: $\mathrm{Xu} T$, Lai X, He K, et al. Subsidy programme for gestational diabetes mellitus screening and lifestyle management in rural areas of western China: a study protocol for a multicentre randomised controlled trial. BMJ Open 2021;11:e045503. doi:10.1136/ bmjopen-2020-045503

- Prepublication history and additional online supplemental material for this paper are available online. To view these files, please visit the journal online. To view these files, please visit the journal online (http://dx.doi.org/10.1136/ bmjopen-2020-045503).

Received 04 0ctober 2020 Accepted 14 June 2021

Check for updates

(c) Author(s) (or their employer(s)) 2021. Re-use permitted under CC BY-NC. No commercial re-use. See rights and permissions. Published by BMJ.

${ }^{1}$ School of Publich Health, Capital Medical University, Beijing, China

${ }^{2}$ School of Public Health, Peking University, Beijing, China

${ }^{3} 0 \&$ G, Peking Union Medical College Hospital, Beijing, China

${ }^{4}$ China Center for Health

Development Studies, Peking University, Beijing, China

Correspondence to

Professor Hai Fang;

hfang@hsc.pku.edu.cn

\section{ABSTRACT}

Introduction Gestational diabetes mellitus (GDM) has become an increasing health problem among pregnant women in western rural China. Insufficient compliance and motivation due to economic factors is one of the major contributors to the currently low GDM screening and management rate. A subsidy program offering GDM screening and lifestyle management might be an effective way to increase pregnant women's awareness of GDM, and further improve maternal and neonatal health in western rural China. This study had two primary purposes: (1) to examine whether the subsidy program would increase the screening and management rates of GDM and reduce adverse complications for mothers and new-born babies and (2) to evaluate whether the subsidy program is cost-effective from a societal perspective.

Methods and analysis This randomised controlled trial will include 3000 pregnant women (at 24-28 weeks of pregnancy) who will be followed up at six hospitals in the provinces of Yunnan, Sichuan and Shaanxi in China. Pregnant women without overt diabetes, with a singleton pregnancy, with telephone access and with written informed consent will be invited. The intervention group will receive subsidies and standard care, and the control group will only receive usual antenatal care. The randomisation sequence will be stratified by study sites with balanced blocks of six patients. Data will be collected using self-report questionnaires and hospital records. Data will be analysed according to the intention-to-treat principle. The primary outcomes are the maternal and neonatal complications. Secondary outcomes are the mother's cognition scores, screening rate, number of reexaminations, weight gain during pregnancy, changes in diet and exercise, and quality of life. Group comparisons will be conducted using $\chi^{2}$ test for categorical variables, and t-test or the Mann-Whitney-Wilcoxon test for continuous variables where applicable. Multiple logistic regression will also be performed for the primary outcomes.

Ethics and dissemination This study was approved by the Ethics Review Committee of Peking University Health Science Center. Findings will be disseminated through publication in peer-reviewed journals, seminars and national and international conferences.

Trial registration number ChiCTR1800017488.
Strengths and limitations of this study

- The study has a long-term follow-up and will provide knowledge on the effects of economic intervention on gestational diabetes mellitus screening and management.

- The study measured the lifestyle (diet, exercise and glucose) and maternal and neonatal compilations as outcomes/effect parameters.

- Pregnant women in western rural China are our participants and the trial's sample size will set up to 3000.

- The nature of the trial interventions prevented blinding of allocation for both medical staff and pregnant women.

- Women in the control group may ask for standard care from providers, which might reduce the effect of this intervention study due to the intention-totreat principle that will be used for data analyses.

\section{INTRODUCTION}

Gestational diabetes mellitus (GDM) is defined as hyperglycaemia diagnosed in the second or third trimester of pregnancy without overt diabetes prior to gestation. ${ }^{1}$ GDM has become an increasing public health problem among pregnant women in China. In 2018, over 2 million pregnant women were affected by hyperglycaemia, and half of cases were found in rural areas. ${ }^{23}$ As China has abolished The One-children Policy, more middle-aged women will get pregnant in the coming future with the potential to increase the incidence of GDM countrywide. Recent studies have estimated that the prevalence of GDM is as high as $20.9 \%$ in several provinces based on the diagnostic criteria of the International Association of Diabetes and Pregnancy Study Groups (IADPSG). ${ }^{45}$

Previous epidemiological studies have reported a significant association between 
GDM and adverse maternal and offspring outcomes. ${ }^{6-8}$ Women with GDM are more susceptible to preeclampsia, preterm birth and macrosomia, ${ }^{9}$ as well as type 2 diabetes after childbirth. ${ }^{10} 11$ Exposure to hyperglycaemia during in utero development is also associated with short-term complications such as macrosomia and neonatal hypoglycaemia, ${ }^{12}$ and in the longer term, increased risks of developing type 2 diabetes or metabolic syndrome. ${ }^{13-15}$

An effective intervention is critical for women with GDM. Studies have indicated that most women with GDM $(80 \%-90 \%)$ can be successfully treated with strict lifestyle management (dietary and/or exercise), reducing the risk of adverse maternal and new-born health outcomes related to GDM, such as caesarean section, macrosomia, preterm birth and neonatal hypoglycaemia. ${ }^{61617}$ In 2011, the Department of Obstetrics and Gynaecology and the Department of Perinatal Medicine in the Chinese Medical Association developed guideline for the diagnosis and treatment of GDM. ${ }^{18}$ This guideline recommended that all pregnant women should receive diagnostic testing with a $75 \mathrm{~g}$ oral glucose tolerance test (OGTT) at 24-28 weeks of gestation and that medical institutions should provide health education regarding GDM to pregnant women. For those diagnosed with GDM, a diet and exercise intervention (lifestyle management) for 2 weeks should be provided. If lifestyle management failed, other treatments, such as insulin or metformin, are suggested to be used depending on individual characteristics.

However, the rates of GDM screening and lifestyle management in western rural China are low. In 2018, the GDM incidence rate increased to $14.5 \%$ in western China, ${ }^{19}$ but the rate of GDM screening and lifestyle management was less than $20.0 \% .^{20} 21$ This situation not only caused a heavy disease burden for women living in undeveloped areas but also posed an economic burden on their families. Our previous study showed that the average cost of treatment for GDM complications was Chinese $¥ 2256$ (US\$1 approximately equal to $¥ 6.23$ in 2015), and the annual societal economic burden of GDM was estimated to be $¥ 19.4$ billion in 2015 . $^{22}$ Thus, efforts to improve screening and lifestyle management and control the potential risk of GDM complications are essential in rural areas of China.

Our pilot investigation in western rural areas show that insufficient compliance is an important cause of the currently low GDM screening and management rate. ${ }^{23}$ Pregnant women have insufficient motivation to receive GDM screening and lifestyle management (diet and exercise management), and have low levels of compliance with medical staff recommendation. Medical staff also have low motivation to provide GDM screening and management since some GDM services are beyond their routine responsibility. Pregnant women living in rural areas usually have lower socioeconomic status and GDM cognition, and they might be unaware of the adverse consequences of GDM. ${ }^{24}{ }^{25}$ Studies also show that a large proportion of pregnant women in rural areas are unwilling to receive or pay for GDM screening and management. ${ }^{26} 27$ The compliance rate with GDM screening and lifestyle management is low in current rural China. Another barrier for the low GDM screening and management rate is the lack of incentives and motivation for medical staff to provide high-quality GDM screening and lifestyle management in rural hospitals since the workload of GDM management usually beyond their routine antenatal services. In contrast, urban hospitals charge extra consulting and/or management fees for providing GDM screening and management, and these hospitals have higher rates of GDM screening and treatment. $^{28}$

The problem of GDM in rural areas is more than merely clinical, it is multifaceted, and requires an approach that reflects this. The majority of previous intervention studies on improving pregnancy outcomes of GDM women explore clinical interventions, including standard care (health education, diet and exercise, and medicine) and single interventions (health education/ diet and exercise/medicine).$^{29}{ }^{30}$ Few studies consider the effect of economic incentives on managing GDM, which is critical for practices on GDM management. Therefore, we propose that a subsidy programme offering GDM screening and lifestyle management in rural areas might substantially promote people's motivation to receive GDM screening and lifestyle management and therefore improve maternal and neonatal health in rural China.

This paper describes the development of this subsidy programme and study procedures.

\section{METHODS AND ANALYSIS}

\section{Study aims}

The main aims of the trial include as follows:

1. To evaluate the effects of a subsidy programme on improving the rate of GDM screening and lifestyle management in western rural China.

2. To determine whether the subsidy programme reduces maternal and new-born complications related to GDM in western rural China.

3. To evaluate whether the subsidy for GDM screening and lifestyle management in rural China is costeffective from a societal perspective.

Additional aims include the following:

1. To determine whether subsidy programmes improve participants' diet and/or exercise.

2. To examine whether subsidised GDM screening and lifestyle management improves pregnant women's understanding of GDM and their quality of life during pregnancy.

\section{Patient and public involvement}

The results of the study will be communicated to participants through a popular scientific report. No other patient involvement has been included. 


\section{Study setting and ethics approval}

This protocol includes the elements elaborated in the Standard Protocol Items: Recommendations for Interventional Trials (SPIRIT) checklist. ${ }^{31}$ Participants will be recruited from the outpatient pregnancy check-ups at six county-level hospitals in three provinces in western China. They are Ziyang Maternal and Child Health Hospital and Hanyin Maternal and Child Health Hospital (in Shaanxi Province), Pingchang Maternal and Child Health Hospital and Yingshan Maternal and Child Health Hospital (in Sichuan Province), Zhaoyang People's Hospital and Yiliang County People's Hospital (in Yunnan Province).

This study was approved by the Peking University Institutional Review Board (registration number: IRB0001052-18052).

\section{Study participants}

Participants will be assessed by asking them whether they are at 24-28 weeks of gestation and whether they will give birth at the study hospital. If the answers to both questions is yes, they will be invited to participate in this project as long as they meet the following inclusion criteria: (1) without overt diabetes before or during pregnancy (ie, type 1 diabetes or type 2 diabetes); (2) with singleton pregnancy; (3) with telephone access from family members, friends or neighbours and (4) with signed informed consent forms (online supplemental file $1)$.

The exclusion criteria are as follows: (1) fasting blood glucose $>7 \mathrm{mmol} / \mathrm{L}$ or glycosylated haemoglobin $\geq 6.5 \%$ at the first GDM screening; (2) concomitant severe systemic diseases (ie, essential hypertension, renal disease, thalassemia, systemic lupus erythaematosus, coeliac disease and thyroid disease) and (3) physical or cognitive disability.

Participants will receive an explanation of the of the study and what would be involved. Written consent will be obtained from all those who express and interest in taking part. Women who do not meet the inclusion criteria will be thanked for their time, and the reasons for exclusion will be documented.

\section{Study design}

Randomisation and blinding

We will perform a stratified multicentre, randomised controlled trial in rural areas of western China. Eligible women who provide consent will undergo an internetbased, computer-generated randomisation procedure incorporating concealed allocation to one of two arms:

1. The intervention group will receive subsidised standard care. The subsidy includes free GDM screening and lifestyle management for women and economic compensation for the medical department. Standard care includes health education, GDM screening and standardised lifestyle management (diet and exercise management).

2. The control group will receive usual care, and pregnant women will receive standard care (health education, GDM screening and standardised lifestyle management) as indicated for the intervention group. However, due to insufficient motivation and compliance among pregnant women and medical staff, there is a large gap between practices and standard care. Therefore, we will use the usual care group instead of the standard care group as the control group.

The randomisation sequence will be stratified by study sites with balanced blocks of six patients and will be held by the ResMan management platform of the China Clinical Trials Registry. An outpatient nurse will perform the allocation. The nature of the trial interventions will prevent blinding of allocation for both medical staff and pregnant women.

\section{Sample size calculation}

The intervention group and usual care group will have the same sample size $(1: 1)$. We calculated that a total sample size of 3000 women (allowing for $20 \%$ drop-out) would provide at least $80 \%$ power to detect a clinically important $25 \%$ increase in the rate of GDM screening from $50 \%$ (observed in the pilot study of 309 women) to $80 \%$. From a review of published population complications in Chinese women with gestational diabetes, 3000 women provided $80 \%$ power to detect a $40 \%$ relative risk reduction for caesarean section (from $51.4 \%$ to $30.5 \%)^{1930}$ and an $80 \%$ relative risk reduction for macrosomia (from $6.2 \%$ to $1.2 \%$ ). ${ }^{30} 32$

\section{Procedure}

Intervention group (subsidised standard care)

The characteristics, knowledge of GDM, diet, physical activities and quality of life of women allocated to the intervention group will be collected with questionnaire 1 (online supplemental file 2), and these women with be required to receive a $\geq 10 \mathrm{~min}$ individual health education session at the beginning of recruitment. This session will provide these women with information on the GDM concept, risk factors, complications, screening procedure, dietary and exercise interventions, recommended foods and recipes, physical activity and treatments. A handbook with the same content will also be provided. Women will be informed and recommended to receive free of charge GDM screening during the sessions. The medical departments that provide the health education sessions will be subsided with $¥ 10$ per participant they persuaded to attend the session. For women rejecting GDM screening, we will document their reasons.

Participants in the intervention group will receive free GDM screening, and an OGTT $(75 \mathrm{~g})$ will be administered to all women between 24 and 28 weeks of gestation using the diagnostic criteria recommended by the IADPSG, namely, fasting venous glucose of $5.1 \mathrm{mmol} / \mathrm{L}$ or higher, 1-hour venous glucose of $10.0 \mathrm{mmol} / \mathrm{L}$ or higher, 2-our venous glucose of $8.5 \mathrm{mmol} / \mathrm{L}$ or higher or a combination of these. ${ }^{33}$

For women diagnosed with GDM in the intervention group, a free of charge $30 \mathrm{~min}$ individual session on diet and exercise management and antenatal retest 
of glucose will subsequently be provided every 2 weeks until their glucose becomes normal. A nurse or doctor will be required to provide remote consultation and management of diet and exercise to women with GDM to promote the effect of self-monitoring every week by WeChat, and a $\log$ book will be used to record their daily diets and exercise (online supplemental file 3), which is useful to help doctors tailor advice for each woman with GDM at retest. If a participant misses her retest, a nurse or doctor will contact and inform her again via telephone or WeChat. Up to three free retests for GDM will be provided according to women's gestational weeks. The medical department was subsidised with $¥ 20$ per participant for the provision of the GDM lifestyle management. For women without GDM, the remote consultation and management of diet and exercise will be provided every week by WeChat in case of abnormal glucose in the last trimester.

Individual recommendations for diet and exercise will be based on the guidelines of diet and exercise for women with GDM, ${ }^{34}$ and will also be adjusted according to participants' habitual preferences. Dietary recommendations will be based on the following principles: restricting dietary intake of saturated fat and exchanging carbohydrate-rich foods with a medium-to-high glycaemic index for foods with a lower glycaemic index to reduce the glycaemic load. For advice on physical activity, ${ }^{34}$ we will focus on incremental increases in walking tailored to the woman's pre-existing activities. Moderate-intensity activities will be suggested, and additional options, including aerobics, yoga and swimming, will be encouraged, particularly for women already engaging in some physical activities. A medical record (online supplemental file 4) will also be used to record the effect of subsidised GDM screening and lifestyle management at delivery. After 34 weeks of gestation, all women in the intervention group will be surveyed again. Data on knowledge of GDM, diet, physical activities and quality of life will be collected via questionnaire 2 (online supplemental file 5).

\section{Control group (usual care)}

All participants allocated to the control group will receive usual care. Additional services, including heath education, screening persuasion, retest information and lifestyle management, will not be required or compensated. We will not provide subsidies for women or medical departments. A medical record will also be used to record the process and results of usual care. At recruitment and after 34 weeks of gestation, all women in this group will be surveyed. Data on the understanding of GDM, diet, physical activities and quality of life will be collected via questionnaires 1 and 2 (online supplemental files 1 and 4 ).

\section{Data collection}

Indexes

To assess the efficacy of subsidised standard care, we have identified 11 effect indexes based on our hypothesis, namely maternal screening rate, cognition for GDM,
Table 1 Maternal and neonatal complications related to GDM

\begin{tabular}{|c|c|}
\hline Maternal complication & $\begin{array}{l}\text { Fetal/neonatal } \\
\text { complication }\end{array}$ \\
\hline Spontaneous abortions & Stillbirth \\
\hline Pre-eclampsia & Neonatal death \\
\hline Gestational hypertension & $\begin{array}{l}\text { Non-chromosomal } \\
\text { congenital malformations }\end{array}$ \\
\hline Amniotic fluid turbidity & Fetal macrosomia \\
\hline Polyhydramnios & Low-birth-weight infants \\
\hline Oligohydramnios & Neonatal asphyxia \\
\hline Intrauterine infection & Neonatal pneumonia \\
\hline Fetal growth restriction & Neonatal anaemia \\
\hline Placenta previa & Neonatal jaundice \\
\hline Preterm labour & Neonatal hypoglycaemia \\
\hline Postmature delivery & Neonatal infection \\
\hline Caesarean delivery & Fetal distress \\
\hline $\begin{array}{l}\text { Premature rupture of } \\
\text { membranes }\end{array}$ & $\begin{array}{l}\text { Neonatal thyroid } \\
\text { abnormalities }\end{array}$ \\
\hline Dystocia & Neonatal cholestasis \\
\hline Postpartum haemorrhage & \\
\hline
\end{tabular}

GDM, gestational diabetes mellitus.

number of retests, dietary and physical activity, gestational weight gain, antenatal visits, quality of life, maternal and neonatal outcomes, and costs in both groups. All maternal and neonatal outcomes are defined by standard clinical diagnosis. These indexes are categorised by primary outcomes and second outcomes.

\section{Primary effectiveness outcomes}

Primary outcomes are adverse complications in mothers and new-born babies related to GDM. We have identified 12 types of GDM-related adverse complications for mothers and new-born babies in the literature (table 1). Among them, five commonly reported maternal complications included hypertensive disorders, premature delivery, postpartum haemorrhage, premature rupture of membranes and caesarean section. Five reported neonatal complications include macrosomia, neonatal jaundice, neonatal pneumonia, neonatal asphyxia and fetal distress in utero. Caesarean section and macrosomia are the most frequently observed complications according to previous studies. ${ }^{19} 32$

\section{Primary cost-effectiveness outcomes}

The primary cost-effectiveness will be measured by the incremental cost per unit decrease in the maternal and/or new-born complication rate between the intervention and control groups. The costs of the proposed GDM subsidy programme include materials, equipment, labour, time, and transportation from a societal perspective. The effectiveness of the proposed GDM subsidy programme indicates avoidable maternal and new-born complications. 
The costs and effectiveness will be compared, and we will examine whether the incremental cost per unit reduction in the complication rate is less than the treatment cost of complications compared with the control group.

\section{Secondary effectiveness outcomes}

Secondary outcomes include the maternal screening rate, cognition for GDM, number of retests, dietary and physical activity, gestational weight gain, antenatal visits and the quality of life measured by the WHO Quality of Life Brief Version (WHOQOL-BREF) ${ }^{35}$

Diet and physical activity will be assessed with the food questionnaire adapted from the Food Frequency Questionnaire (FFQ) of pregnant women in rural China ${ }^{36-39}$ and the physical activity questionnaire is a simplified version of the self-report physical activity scale from the International Physical Activity Questionnaire (IPAQ). ${ }^{40} 41$ We will exclude participants who are estimated to be underreporting (weight of food intake $\leq 5 \mathrm{~g}$ per time) or overreporting (weight of food intake $\geq 10 \mathrm{~kg}$ per time) their food intake.

\section{Questionnaire}

To collect the data in this study, we will use two questionnaires that include five types of instruments, a medical record and a cost form.

\section{Questionnaire 1}

1. The basic information portion consists of two sections: demographic characteristics and a self-designed cognitive scale of GDM.

Demographic and sociological characteristics include age, height, weight, weeks of pregnancy, blood pressure, parity, family disease history, residence, education level and occupational status, among other factors. Family economic situation refers to household resident population, personal income, family income and medical insurance, among other factors. Transportation comprises transportation time, cost and lost working days, among others, to calculate direct non-medical intervention costs and indirect intervention costs in the health economics evaluation.

The cognition portion is comprised of nine 5-point scale questions. The higher the score is, the better the cognition is. We will calculate a total cognitive score by multiplying the average score of each item by 4 and then converting the score into the percentage system according to the following formula: total score $=($ total score - 4$) *(100 / 16)$.

2. Diet and physical activity. Changes in diet and physical activity from baseline to 34-36 weeks of gestation were measured by a modified FFQ and self-reported physical activity scale from the IPAQ.

- FFQ: This part includes 15 categories according to the recommended classification of food and supplements of China's GDM guidelines: rice, wheat flour, cereals, potato flour, fried food, dark vegetables, fresh fruits, poultry/livestock meat, aquatic prod- ucts, eggs, milk and its products, beans and their products, nuts, snacks and beverages. The nutritional components (energy, carbohydrate, protein, fat, fibre) are measured accordingly.

- Physical activity: This part was designed to investigate the frequency and duration of vigorous exercise, moderate exercise and walking among pregnant women.

3. Quality-of-life scale. Each woman's quality of life will be measured via the WHOQOL-BREF at the beginning of recruitment and at 34-36 weeks of gestation. There are four dimensions, namely, physical health, psychological health, social relationships and the environment, with a total of 26 questions. The WHOQOL-BREF is commonly used to measure quality of life over 2 weeks with great reliability and validity. The score of each dimension is recorded in a positive way and is obtained by calculating the average score of each item and multiplying it by 4 according to the following formula: domain score $=($ score- 4$) *(100 / 16)$.

\section{Questionnaire 2}

Questionnaire 2 adds a section of examination items and the costs for women with GDM. These items include urinalysis, glucose tests, the glycated albumin test, the glycosylated, haemoglobin test, b-mode ultrasonography, liver and kidney function examinations, insulin levels and other. Two questions were added to the section on basic information, which includes antenatal visits, the average cost per visit, the total cost, and the total out-of-pocket cost. The component on cognition, diet and physical activity is the same as in questionnaire 1 .

\section{Medical records}

Medical records were designed for nurses to record the effect of the intervention on pregnant women, including on the screening rate, blood glucose value, lifestyle management rate, GDM re-examination rate and outcomes of mothers and new-born babies. Maternal and new-born complications will be diagnosed by standard clinical criteria.

- GDM screening: Information on the fasting blood glucose value, 1-hour glucose and 2-hour glucose levels of the $75 \mathrm{~g}$ OGTT, screening rate, number of re-examinations, and whether the patient received a diet and exercise session will be collected. The performance of nurses and doctors will also be measured at this stage.

- GDM retests pregnant women with GDM will be required to have one to three re-examinations based on their gestational weeks. Fasting blood glucose and 2-hour glucose levels after a meal will also be recorded.

- Maternal complications include caesarean section, premature delivery (defined as gestational weeks at birth $<37$ weeks), gestational hypertension (defined as systolic blood pressure $\geq 140 \mathrm{~mm} \mathrm{Hg}$, diastolic blood pressure $\geq 90 \mathrm{~mm} \mathrm{Hg}$ or both on at least two occasions 4 hours apart, with proteinuria $\geq 300 \mathrm{mg} / 24$ hours, 
spot urine protein showing creatinine ratio $\geq 30 \mathrm{mg} /$ mmol creatinine or urine dipstick protein $\geq 2+$ ), postpartum haemorrhage and premature rupture of membranes, among others.

- Neonatal complications include fetal macrosomia (birthweight $\geq 4.0 \mathrm{~kg}$ ), neonatal jaundice, pneumonia of the new-born, pneumonia asphyxia and fetal distress in the uterus.

\section{Form on costs}

Information of direct intervention costs and indirect intervention costs will be collected from a social perspective. Direct intervention costs are categorised into direct medical costs and direct non-medical costs.

- Direct medical costs include the GDM screening cost, consultation and management cost, GDM re-examination cost and additional examination cost. The unit cost of each category will be estimated based on market price. We will not measure the costs of insulin treatment for pregnant women with GDM. Transportation costs will be regarded as direct non-medical costs in this study.

- Indirect intervention costs include the cost of missed working days for women and their families according to the minimum hour wage in 2018 and garbage disposal cost.

\section{Statistical analysis}

Data will be entered using Epidata software, and statistical analyses will be carried out using STATA V.16.0 (Stata). Bivariate analyses (t-tests or the Mann-Whitney-Wilcoxon test for continuous measures and $\chi^{2}$ tests for categorical measures) and multivariate analyses (ordinary least squares regression, logistic regression, generalised linear estimation and others) will be employed to estimate the effects (control for confounding variables) of subsidies for GDM screening and lifestyle treatment in rural China. Repeated data will be examined using difference-indifference regression models. A general growth mixture model will be used to test a joint trajectory of female characteristics at pregnancy and GDM intervention with maternal and child outcomes. Both parametric and nonparametric econometric analyses will be implemented. The costs (implementing the subsidy programme) and effectiveness/benefits for both women and children will be compared, and the incremental cost-effectiveness and cost-benefit ratios will be studied.

To minimise the loss of information and potential bias caused by missing data, we will use multiple imputation to generate plausible values for each missing value of covariables in our final models. We will generate 50 completed data sets for each set of missing data using STATA.

\section{Ethics and dissemination}

Ethics approval and consent to participate

Ethical approval was obtained from the Ethics Review Committee of Peking University Health Science Center in Beijing China (PU IRB reference code: IRB00001052-18052).

\section{Informed consent}

Written informed consent will be obtained from pregnant women in the pre- and post-intervention study prior to participant involvement in the study. The consent form

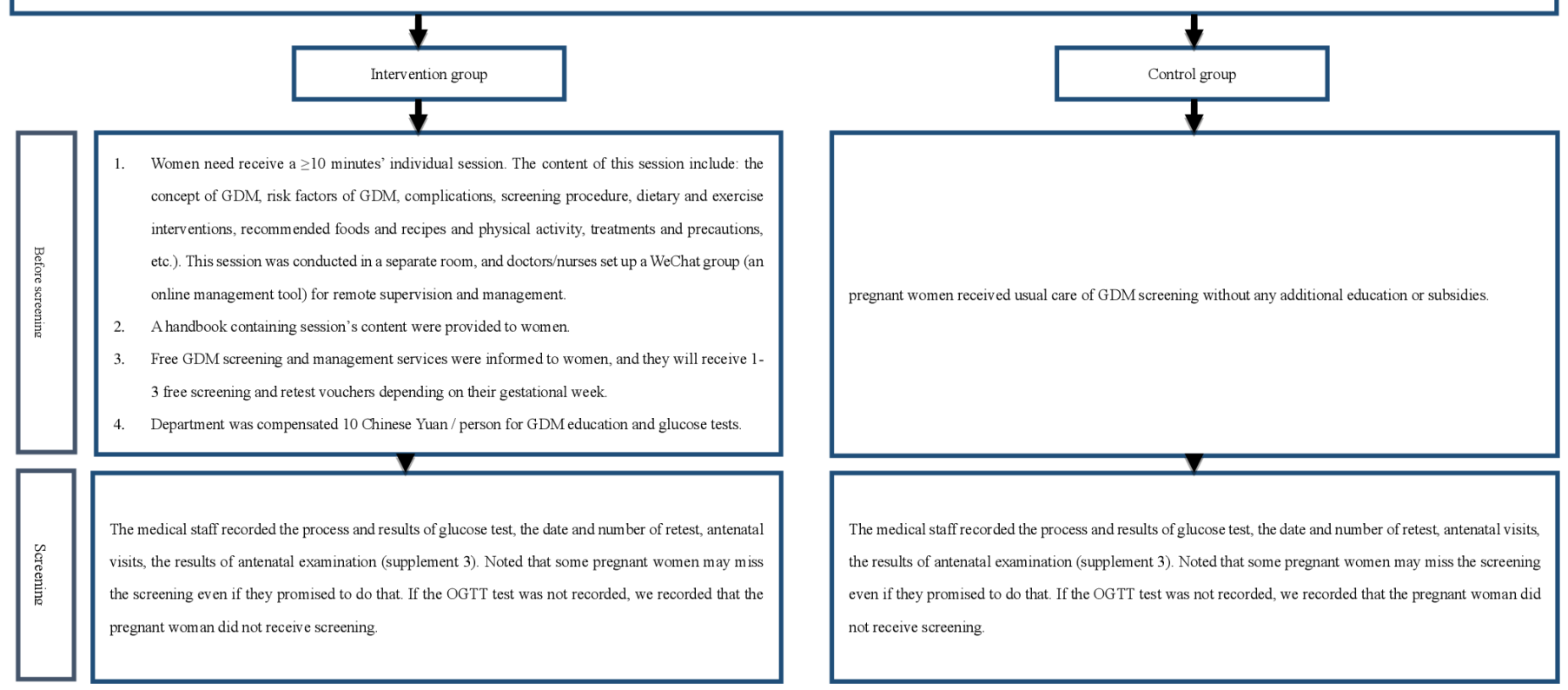

Figure 1 Flow chart of participants' selection. GDM, gestational diabetes mellitus; OGTT, oral glucose tolerance test. 


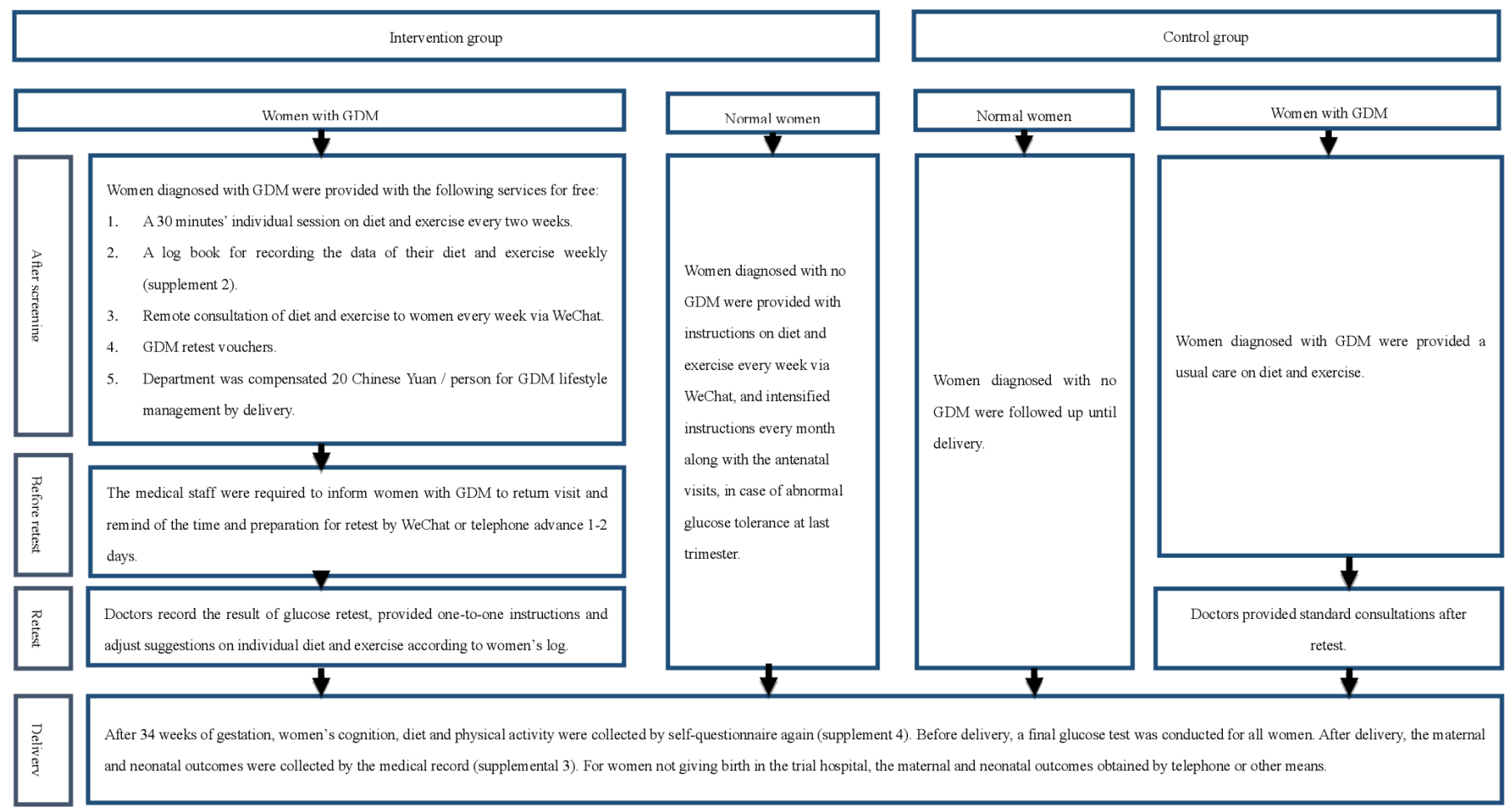

Figure 2 Flow chart (continued). GDM, gestational diabetes mellitus.

is held by institutions (Peking University) and is available for review from the Chief Investigator.

\section{Data management}

Data from this study will be stored on an electronic server (ResMan management system) at the platform of the China Clinical Trials Registry. Written informed consent forms, questionnaires, medical records and cost forms will initially be stored in a locked storeroom at the hospital and then delivered by a nurse to the Department of Public Health at Peking University. Personal data that can identify individuals will be deleted at the beginning of 2021, but anonymous information will be used for follow-up studies, depending on future funding.

\section{Dissemination plan}

In our study, we followed the World Medical Association Declaration of Helsinki (http://www.wma.net). Women meeting the inclusion criteria will be informed that neither the decision to participate nor decline of participation will influence their care. Participation is voluntary, and women can withdraw whenever they want.

Medical staff have been made aware of this. The intervention is a non-invasive method. The $75 \mathrm{~g}$ OGTT used in this study has certificate approval.

This study has been considered by the Ethics Review Committee of Peking University Health Science Center and the patient privacy protection boards governing the recruitment sites.

Important modifications to the protocol are updated on the ClinicalTrials.gov website and disseminated to all relevant parties. We have a contractual agreement with key collaborators determining access to trial data and authorship.

\section{Data services}

Sensitive information such as the participant's name, project ID number, phone number, country of birth from the recruitment form and information from the medical records will be stored in a locked cupboard at the hospitals. Every person involved in the recruitment has signed a confidentiality form. The results from the study will be presented in scientific journals and at national and international congresses.

\section{DISCUSSION}

GDM is the leading attributable risk factor for some serious complications that occur in women during pregnancy. It is estimated that the incidence rate of GDM has been increasing across China, and by 2019, approximately 3 million pregnant women suffered from GDM in China. ${ }^{45}$ Despite many studies exploring interventions to reduce adverse consequences among women with GDM,${ }^{17} 42$ few studies have tried to study the features of GDM in rural areas, which are different from those in urban areas. This exploratory research can provide a new direction for improving health quality and equity in rural areas.

At present, various GDM interventions and management strategies focusing on improving women's compliance aimed to attract pregnant women by designing new methods of management. There is a lack of incentive to encourage women to seek GDM screening and treatment 
initially. Additionally, health providers' demands are usually ignored, while in practice, a large proportion of personnel in GDM management services are beyond their regular clinical workload. In this study, we propose subsidised GDM screening and management for pregnant women and medical staff. We supposed that standard care together with economic intervention would substantially increase their motivation. In addition, once women are diagnosed with GDM, the expenses of GDM screening and treatment are paid completely out of pocket in China, which is a large obstacle for women with low income in terms of receiving appropriate care. Subsidised GDM care that is provided for women and medical staff is essential for the health improvement of individuals with insufficient initiative.

In this study, we offered subsidies to two types of GDM services: screening and lifestyle management. This study will help us to determine which means of financial intervention has better performance. We are not yet sure who is the largest contributor to the low screening rate in rural areas: pregnant women or healthcare providers. Separate subsidies on GDM screening and management can help make this distinction.

Our outcomes not only evaluate the effectiveness of subsidised standard care intervention for women in western rural China but also show the cost-effectiveness of the intervention. The results of the cost-effectiveness analysis will demonstrate whether our project has economic feasibility in the future for popularisation and application. Another point is that we only provide subsidy to women in the intervention group. There is no contamination between the two groups.

\section{Trial status}

Participants are being recruited. The first patient was enrolled in September 2018. The SPIRIT study timeline details are shown in figures 1 and 2.

Acknowledgements We would like to acknowledge the members of the six hospitals and their directors, doctors and nurses for recruitment and data collection. The members include Ping Zhang, Min Fan, Yincui Wang, Chunmei Yin, Xinmei Xiang, Qingyan Li, Hanli Zhang, Renping Zhang, Mengfan Ma and Xiaohuan Tang. The authors would like to thank the pregnant women who are participating in the feasibility study and the ongoing RCT study.

Contributors HF conceptualised this study, contributed to the interpretation of the results and reviewed and substantially revised the manuscript. TX conceived the study question and designed the study, conducted the data analyses and wrote the first draft of the manuscript. XL and KH reviewed and edited the manuscript substantially. LM contributed to the interpretation of the results and critically reviewed and edited the manuscript for important intellectual content. All authors approved the final manuscript as submitted and agree to be accountable for all aspects of the work.

Funding The study is supported by a research award: China Medical Board Grant Number 17-266.

Disclaimer The funders had no role in the study design, data collection and analysis, decision to publish or preparation of the manuscript.

Competing interests None declared.

Patient consent for publication Not required.

Provenance and peer review Not commissioned; externally peer reviewed.
Supplemental material This content has been supplied by the author(s). It has not been vetted by BMJ Publishing Group Limited (BMJ) and may not have been peer-reviewed. Any opinions or recommendations discussed are solely those of the author(s) and are not endorsed by BMJ. BMJ disclaims all liability and responsibility arising from any reliance placed on the content. Where the content includes any translated material, BMJ does not warrant the accuracy and reliability of the translations (including but not limited to local regulations, clinical guidelines, terminology, drug names and drug dosages), and is not responsible for any error and/or omissions arising from translation and adaptation or otherwise.

Open access This is an open access article distributed in accordance with the Creative Commons Attribution Non Commercial (CC BY-NC 4.0) license, which permits others to distribute, remix, adapt, build upon this work non-commercially, and license their derivative works on different terms, provided the original work is properly cited, appropriate credit is given, any changes made indicated, and the use is non-commercial. See: http://creativecommons.org/licenses/by-nc/4.0/.

ORCID iDs

Tingting Xu http://orcid.org/0000-0001-5507-3061

Liangkun Ma http://orcid.org/0000-0001-5758-0198

\section{REFERENCES}

1 American Diabetes Association. 13. Management of diabetes in pregnancy: Standards of Medical Care in Diabetes-2018. Diabetes Care 2018;41:S137-43.

2 Zhu W-w, Yang H-x, Wei Y-m, et al. Evaluation of the value of fasting plasma glucose in the first prenatal visit to diagnose gestational diabetes mellitus in China. Diabetes Care 2013;36:586-90.

3 National Health and Family Planning Commission of China. China's health and family planning statistical yearbook 2015. Beijing: Peking Union Medical College Press, 2015.

4 Wu L, Han L, Zhan Y. Prevalence of gestational diabetes mellitus and associated risk factors in pregnant Chinese women: a crosssectional study in Huangdao, Qingdao, China. Asia Pac J Clin Nutr 2018;27:383-8

5 Wu J-N, Gu W-R, Xiao X-R, et al. Gestational weight gain targets during the second and third trimesters of pregnancy for women with gestational diabetes mellitus in China. Eur J Clin Nutr 2019;73:1155-63.

6 Johns EC, Denison FC, Norman JE, et al. Gestational diabetes mellitus: mechanisms, treatment, and complications. Trends in Endocrinology \& Metabolism 2018;29:743-54.

7 Petry CJ. Gestational diabetes: origins, complications, and treatment Boca Raton: Taylor \& Francis/CRC Press, 2014.

8 The HAPO Study Cooperative Research Group. Hyperglycemia and adverse pregnancy outcomes. N Engl J Med 2008;2008:1991-2002.

9 Kanguru L, Bezawada N, Hussein J, et al. The burden of diabetes mellitus during pregnancy in low- and middle-income countries: a systematic review. Glob Health Action 2014;7:23987.

10 Poomalar GK. Changing trends in management of gestational diabetes mellitus. World J Diabetes 2015;6:284-95.

11 Verier-Mine O. Outcomes in women with a history of gestational diabetes. screening and prevention of type 2 diabetes. literature review. Diabetes Metab 2010;36:595-616.

12 Kitzmiller JL, Dang-Kilduff L, Taslimi MM. Gestational diabetes after delivery: short-term management and long-term risks. Diabetes Care 2007;30:S225-35.

13 Ehrlich SF, Rosas LG, Ferrara A, et al. Pregnancy glycemia in Mexican-American women without diabetes or gestational diabetes and programming for childhood obesity. Am J Epidemiol 2013;177:768-75.

14 Lowe WL, Scholtens DM, Lowe LP, et al. Association of gestational diabetes with maternal disorders of glucose metabolism and childhood adiposity. JAMA 2018;320:1005-16.

15 Gingras V, Rifas-Shiman SL, Derks IPM, et al. Associations of gestational glucose tolerance with offspring body composition and estimated insulin resistance in early adolescence. Diabetes Care 2018;41:e164-6.

16 Falavigna M, Schmidt MI, Trujillo J, et al. Effectiveness of gestational diabetes treatment: a systematic review with quality of evidence assessment. Diabetes Res Clin Pract 2012;98:396-405.

17 Viana LV, Gross JL, Azevedo MJ. Dietary intervention in patients with gestational diabetes mellitus: a systematic review and meta-analysis of randomized clinical trials on maternal and newborn outcomes. Diabetes Care 2014;37:3345-55.

18 Yang $\mathrm{H}$. Guidelines for diagnosis and treatment of gestational diabetes mellitus (2014 edition). Chinese Journal of Obstetrics and Gynecology 2014;49:561-9. 
19 Mak JKL, Lee AH, Pham NM, et al. Gestational diabetes incidence and delivery outcomes in Western China: a prospective cohort study. Birth 2019;46:166-72.

20 Wang $\mathrm{H}$, Zhang $\mathrm{Y}$. Study on the high risk factors and pregnancy outcomes of gestational diabetes mellitus in rural areas. Chinese Maternal and Child Health Research;201:59-63.

21 Luo S. Influence factors of gestational diabetes mellitus in rural women of childbearing age. New World of Diabetes Mellitus 2016;16:72-3.

22 Xu T, Dainelli L, Yu K, et al. The short-term health and economic burden of gestational diabetes mellitus in China: a modelling study. BMJ Open 2017;7:e018893.

23 Lin T. Influence of standardized treatment on pregnancy outcomes in pregnant women with diabetes mellitus. Chinese Pharmaceutical Economics 2015;7:98-9.

24 Fang J. Study on the mental status of pregnant women and its influencing factors. Chinese Psychological Society 2009;1:1-14.

25 Lin F. Investigation and analysis of mental health status of pregnant women. Clinical Medical Engineering 2013;11:1453-4.

26 Zhang Y, Li X. Under the new situation to further reform and improve. Collection of Women's Studies, Maternity Insurance System 2015;6:41-6.

27 Tan $\mathrm{C}$, Zhang $\mathrm{H}$. Evolution of China's maternity insurance system and government responsibility. Chinese Soft Science 2011;8:14-20.

28 Chinese Price Bureaus. Basic medical service prices in Beijing; 2015: 1-7. http://fgw.beijing.gov.cn/so/s?tab=all\&siteCode= 1100000011

29 Agha-Jaffar R, Oliver N, Johnston D, et al. Gestational diabetes mellitus: does an effective prevention strategy exist? Nat Rev Endocrinol 2016;12:533-46.

$30 \mathrm{Xu} \mathrm{T}, \mathrm{He}$ Y, Dainelli L, et al. Healthcare interventions for the prevention and control of gestational diabetes mellitus in China: a scoping review. BMC Pregnancy Childbirth 2017;17:171.

31 Chan A-W, Tetzlaff JM, Gotzsche PC et al. Spirit 2013 explanation and elaboration: guidance for protocols of clinical trials. BMJ 2013;346:e7586.
32 Wang D, Hong Y, Zhu L, et al. Risk factors and outcomes of macrosomia in China: a multicentric survey based on birth data. The Journal of Maternal-Fetal \& Neonatal Medicine 2017;30:623-7.

33 Metzger BE, Gabbe SG, Persson B, et al. International association of diabetes and pregnancy study groups recommendations on the diagnosis and classification of hyperglycemia in pregnancy: response to Weinert. Diabetes Care 2010;33:e98-682.

34 Yang $\mathrm{H}, \mathrm{Xu}$ X, Wang Z. Guidelines for diagnosis and treatment of gestational diabetes mellitus (2014). Chinese Journal of perinatal medicine 2014;17:537-45.

35 THE WHOQOL GROUP. Development of the world Health organization WHOQOL-BREF quality of life assessment. Psychol Med 1998;28:551-8.

36 Liu D, He L, Zhang X. Establishment and application of food frequency questionnaire method among Chinese. Wei Sheng Yan Jiu 2018;47:744-55.

37 Zhang H, Qiu X, Zhong C. Reproducibility and relative validity of a semi-quantitative food frequency questionnaire for Chinese pregnant women. Nutr J 2015;4:56.

38 Cheng $\mathrm{Y}$, Yan H, Dibley M. Validity and reproducibility of a semiquantitative food frequency questionnaire for use among pregnant women in rural China. Asia Pac J Clin Nutr 2008;17:166-77.

39 Willett W. Food-Frequency methods. In: Willett W, ed. Nutritional epidemiology. 2nd ed. Oxford: Oxford University Press, 1998.

40 Qu N, Li K. Study on the reliability and validity of international physical activity questionnaire (Chinese vision, IPAQ). Zhong hua Liu Xing Bing Xue Za Zhi 2004;25:265-8.

41 Ren Y, Su M, Liu Q. Validation of the simplified Chinese-character version of the International physical activity Questionnaire-Long form in urban community-dwelling adults: a cross-sectional study in Hangzhou, China. Biomed Environ Sci 2017;30:255-63.

42 Martis R, Crowther C, Shepherd E, et al. Treatments for women with gestational diabetes mellitus: an overview of Cochrane systematic reviews. Cochrane Database Syst Rev 2018;8:CD012327. 